\title{
Outcome after aortic valve replacement in children: A systematic review and meta-analysis
}

\author{
Jonathan R. G. Etnel, BSc, ${ }^{\text {a }}$ Lisa C. Elmont, BSc, ${ }^{\mathrm{a}}$ Ebru Ertekin, BSc, ${ }^{\mathrm{a}}$ M. Mostafa Mokhles, MD, PhD, ${ }^{\mathrm{a}}$ \\ Helena J. Heuvelman, MD, PhD, ${ }^{\mathrm{a}}$ Jolien W. Roos-Hesselink, MD, PhD, ${ }^{\mathrm{b}}$ Peter L. de Jong, MD, ${ }^{\mathrm{a}}$ \\ Willem A. Helbing, MD, PhD, ${ }^{c}$ Ad J. J. C. Bogers, MD, PhD, ${ }^{a}$ and Johanna J. M. Takkenberg, MD, PhD ${ }^{\mathrm{a}}$
}

\begin{abstract}
Objective: Despite an increasing interest in pediatric aortic valve repair, aortic valve replacement in children may be unavoidable. The evidence on outcome after pediatric aortic valve replacement is limited and usually reported in small case series. This systematic review and meta-analysis aims to provide an overview of reported outcome of pediatric patients after aortic valve replacement.
\end{abstract}

Methods: A systematic literature search for publications reporting outcome after pediatric aortic valve replacement published between January 1990 and May 2015 was conducted. Studies written in English with a study size of more than 30 patients were included.

Results: Thirty-four publications reporting on 42 cohorts were included in this review: 26 concerning the Ross procedure $(n=2409), 13$ concerning mechanical prosthesis aortic valve replacement $(n=696)$, and 3 concerning homograft aortic valve replacement $(\mathrm{n}=224)$. There were no studies on bioprostheses that met our inclusion criteria. The pooled mean patient age was 9.4 years, 12.8 years, and 8.9 years for Ross, mechanical prosthesis, and homograft recipients, respectively. Pooled mean follow-up was 6.6 years. The Ross procedure was associated with lower early $(4.20 \%$; $95 \%$ confidence interval $[\mathrm{CI}], 3.37-5.22$ vs $7.34 \% ; 95 \%$ CI, 5.21-10.34 vs $12.82 \%$; $95 \%$ CI, 8.91-18.46) and late mortality $(0.64 \% / y$; $95 \%$ CI, $0.49-0.84$ vs $1.23 \% / y$; $95 \%$ CI, $0.85-1.79$ vs $1.59 \% / y$; $95 \%$ CI, 1.03-2.46) compared with mechanical prosthesis aortic valve replacement and homograft aortic valve replacement, respectively. No significantly different aortic valve reoperation rates were observed between the Ross procedure and mechanical prosthesis aortic valve replacement $(1.60 \% / y ; 95 \%$ CI, $1.27-2.02$ vs $1.07 \% / y ; 95 \%$ CI, 0.68-1.68, respectively), whereas homograft aortic valve replacement was associated with significantly higher aortic valve reoperation rates $(5.44 \% / y$; $95 \% \mathrm{CI}, 4.24-6.98)$. The Ross procedure-associated right ventricular outflow tract reoperation rate was $1.91 \%$ per year $(95 \%$ CI, $1.50-2.44)$.

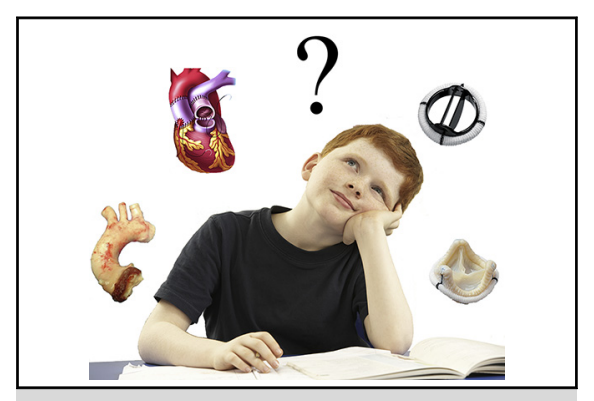

The dilemma of prosthesis choice in pediatric AVR.

Central Message

All valve substitutes provide suboptimal results in children, reflecting the urgent need for innovative repair and replacement options.

\section{Perspective}

The balance of the risks and benefits of the various valve prostheses remains a point of discussion. This systematic review, encompassing a total of 3329 patients with 21,110 patientyears of follow-up, provides a comprehensive overview of the clinical benefits and limitations of the various aortic valve substitutes in children, crucial information to optimize the surgical decision-making process.

See Editorial Commentary page 153

Conclusions: This systematic review illustrates that all currently available aortic valve substitutes are associated with suboptimal results in children, reflecting the urgent need for reliable and durable repair techniques and innovative replacement solutions for this challenging group of patients. ( $\mathbf{J}$ Thorac Cardiovasc Surg 2016;151:143-52)

\footnotetext{
From the Departments of ${ }^{\mathrm{a}}$ Cardiothoracic Surgery and ${ }^{\mathrm{b}}$ Cardiology, Erasmus University Medical Center, Rotterdam, The Netherlands; and the ${ }^{\mathrm{c}}$ Department of Pediatric Cardiology, Erasmus University Medical Center-Sophia Children's Hospital, Rotterdam, The Netherlands.

This work was supported by the Dutch Heart Foundation (2013T093)

The contributions of J.R.G.E., L.C.E., and E.E. are equally acknowledged

Received for publication Nov 13, 2014; revisions received Aug 18, 2015; accepted for publication Sept 15, 2015; available ahead of print Nov 3, 2015.
}

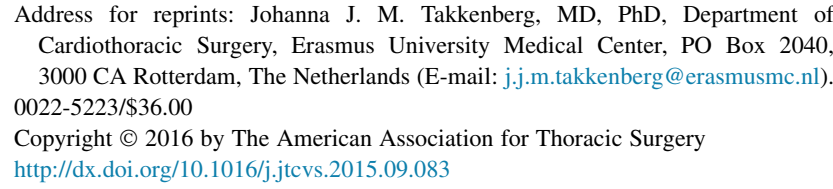

Address for reprints: Johanna J. M. Takkenberg, MD, PhD, Department of Cardiothoracic Surgery, Erasmus University Medical Center, PO Box 2040, 3000 CA Rotterdam, The Netherlands (E-mail: j.j.m.takkenberg@erasmusmc.nl). 0022-5223/\$36.00

Copyright (C) 2016 by The American Association for Thoracic Surgery http://dx.doi.org/10.1016/j.jtcvs.2015.09.083 


$$
\begin{aligned}
& \text { Abbreviations and Acronyms } \\
& \text { AVR = aortic valve replacement } \\
& \text { CI }=\text { confidence interval } \\
& \text { HG }=\text { homograft } \\
& \text { MP }=\text { mechanical prosthesis } \\
& \text { NSVD }=\text { nonstructural valve dysfunction } \\
& \text { NYHA }=\text { New York Heart Association } \\
& \text { PPM }=\text { prosthesis-patient mismatch } \\
& \text { RVOT }=\text { right ventricular outflow tract } \\
& \text { SVD }=\text { structural valve deterioration } \\
& \text { TE }=\text { thromboembolism } \\
& \text { VT }=\text { valve thrombosis }
\end{aligned}
$$

Supplemental material is available online.

Although pediatric aortic valve repair is rapidly developing and meets great interest, aortic valve replacement (AVR) often cannot be avoided. The outcome after AVR in pediatric patients is reported infrequently and usually in small retrospective case series. All currently available surgical options in children have certain limitations, and the choice of valve substitute is determined by several factors. In addition to the occurrence of valve-related complications, ${ }^{1}$ the influence of patient growth has a major impact on valve performance in children. In current clinical practice, 4 types of aortic valve substitutes can be offered to children who require AVR: the Ross procedure, mechanical prostheses (MPs), homografts (HGs), and bioprostheses. The Ross procedure (a pulmonary autograft in the aortic valve position and an allograft in the pulmonary position) is considered the preferred surgical option for children who require AVR. ${ }^{2,3}$ It is the only living valve substitute and has proven to be hemodynamically superior without the need for long-term anticoagulation, shows diameter increase along with somatic growth, and is associated with a low risk of endocarditis. ${ }^{4,5}$ Nevertheless, the Ross procedure is a complex surgical procedure, and both the pulmonary autograft and the valve substitute in the right ventricular outflow tract (RVOT) may require reintervention. ${ }^{6}$ The primary advantage of MPs is long-term performance. However, in addition to bleeding complications due to lifelong anticoagulation, prosthesispatient mismatch (PPM) can cause deterioration of ventricular function in growing children. ${ }^{7-11}$ Furthermore, female patients with mechanical heart valves face a substantial risk of serious complications during future pregnancies. ${ }^{12}$ HGs have a low thrombogenicity and favorable tissue characteristics that allow for complex reconstruction of the aortic root, but have a limited durability because of early calcifications and may not be readily available. ${ }^{8,13,14}$ Bioprostheses have the advantage of commercial availability and assumingly perform similar to HGs; however, high rates of early degeneration, calcification, and structural failure have been reported in young recipients. ${ }^{8,9,15-18}$

The balance of the risks and benefits of the various pediatric AVR alternatives remains a point of discussion, and an overview of reported outcomes is lacking. Therefore, the aim of this systematic literature review and metaanalysis is to provide an overview of the published evidence reporting outcomes after contemporary pediatric AVR with pulmonary autografts, MPs, HGs, and bioprostheses.

\section{MATERIALS AND METHODS Search Strategy}

To establish an overview of published evidence on outcome after pediatric AVR, we conducted a systematic review according to the Preferred Reporting Items for Systematic Reviews and Meta-Analyses guidelines. ${ }^{19}$ On March 19, 2015, PubMed and Embase were searched (Appendix E1). The search was limited to studies that included 30 or more patients, were conducted in humans, were published after January 1, 1990, and were written in English. We also applied a limit on mean patient age $(<18$ years $)$ and maximum patient age $(<21$ years $)$ at the time of surgery. All results were screened for study design and outcome (early and late mortality, reoperations, and complications). Only the most recent or most complete study was included in case study populations were overlapping. A second independent reviewer (MMM) assessed whether inclusion and exclusion were performed correctly. In case of disagreement, an agreement was negotiated. References of selected articles were cross-checked for other relevant studies.

\section{Data Extraction}

Microsoft Office Excel 2011 (Microsoft Corp, Redmond, Wash) was used for data extraction. Publications were categorized by prosthetic valve type: Ross procedure, MP AVR, HG AVR, and bioprosthetic AVR. Studies describing more than 1 type of AVR procedure were included as separate cohorts according to the type of AVR procedure performed. Studies that included only infants and neonates were pooled separately. Year of publication, number of patients, study design, and follow-up (patient-years and mean/median follow-up) were recorded as study characteristics. If follow-up was not reported in patient-years, mean or median follow-up was multiplied by the reported number of patients. The following baseline patient characteristics were recorded: mean age at time of AVR, gender, indication for AVR surgery, previous cardiac interventions, concomitant procedures, and annular enlargement procedures. The indication for surgery was categorized by cause of valve disease (eg, congenital, rheumatic, and endocarditis). Morbidity and mortality were documented according to the guidelines as described by Akins and colleagues. ${ }^{20}$ The following events were documented: early mortality $(\%)$, late mortality (\%/year), reoperation (\%/year), and complications (\%/year). Early mortality was defined as death within 30 days after AVR, and late mortality was defined as death after 30 days postoperatively. All reoperations after initial AVR, including percutaneous interventions, were registered. Reoperations were divided into aortic valve reoperations and for the Ross procedure RVOT reoperations. A distinction was made between all-cause reoperations and reoperations for structural valve deterioration (SVD)/ nonstructural valve dysfunction (NSVD). Reoperations for neoaortic root dilatation/aneurysm after the Ross procedure were recorded as reoperations for SVD/NSVD. The following complications occurring more than 30 days after AVR were documented: thromboembolism (TE)/valve thrombosis (VT), bleeding, and endocarditis. ${ }^{20}$ Functional health status measured by 
the New York Heart Association (NYHA) classification was registered when described in the study.

\section{Statistical Analyses}

Weighted pooled baseline patient characteristics were calculated for each prosthetic valve type group. The Student $t$ test and the chi-square test were used to test for differences in continuous and categoric baseline characteristics, respectively. Early mortality risk and linearized occurrence rates of late mortality, sudden unexpected and unexplained death, reoperations, and complications after AVR were calculated and pooled with the use of inverse variance weighting on a logarithmic scale, because the Shapiro-Wilk test revealed a significantly skewed distribution among the included studies in the majority of outcome measures. When the number of studies was sufficiently large to reliably estimate the tausquared statistic ( $\geq 4$ studies), a random effects model was used to estimate pooled effects. When estimating pooled effects from less than 4 studies, a fixed effects model was used. In case a particular event was reported not to occur in an individual study, then for the purpose of the analyses it was assumed that 0.5 patient experienced an event. The Cochran Q statistic and the $\mathrm{I}^{2}$ test were used to assess heterogeneity. Potential causes of heterogeneity were explored by investigating the effect of year of first inclusion, mean follow-up duration, and case mix. Funnel plots were used to investigate publication bias. Statistical analyses were performed in Microsoft Office Excel 2011 (Microsoft Corp), IBM SPSS Statistics (version 21.0.0.1. IBM Corp, Armonk, NY), and the R statistical software (version 3.1.0. R Development Core Team, R Foundation for Statistical Computing, Vienna, Austria) using the metafor package. The authors had full access to and take full responsibility for the integrity of the data.

\section{RESULTS}

The search resulted in 1218 publications after removal of duplicates. After applying inclusion and exclusion criteria, a total of 34 publications were included in the systematic review, reporting on 42 cohorts: 26 concerning the Ross procedure $(\mathrm{n}=2409),{ }^{11,13,16,21-43} 13$ concerning MP-AVR $(\mathrm{n}=696),{ }^{15,16,23,31,44-52}$ and 3 concerning HG-AVR $(\mathrm{n}=224)^{13,23,53}$ (Figure 1). There were no studies on bioprosthetic AVR that met our inclusion criteria, because all of them had a sample size of less than 20 patients. This systematic review encompasses a total of 3329 patients with 21,110 patient-years of follow-up.

\section{Study Characteristics and Baseline Patient Characteristics}

Table 1 provides an overview of the publications included in the present study. Pooled mean follow-up was 6.6 years. Indication for AVR was mainly congenital heart disease. By excluding studies that included only neonates and infants, the pooled mean age was $9.5 \pm 4.9$ years (range, 5.17-14.0 years) for the Ross procedure, $12.8 \pm 3.6$ years (range, 9.0-16.6 years) for MP-AVR, and $8.9 \pm 4.1$ years (range, 4.9-12.5 years) for HG-AVR.

When comparing patients undergoing the Ross procedure (excluding studies that included only neonates and infants) with patients undergoing MP-AVR, patients undergoing the Ross procedure were significantly younger $(P<.001)$, less frequently had rheumatic valve disease $(19.2 \%$ vs $36.1 \%$, $P<.001$ ), and more frequently had undergone previous

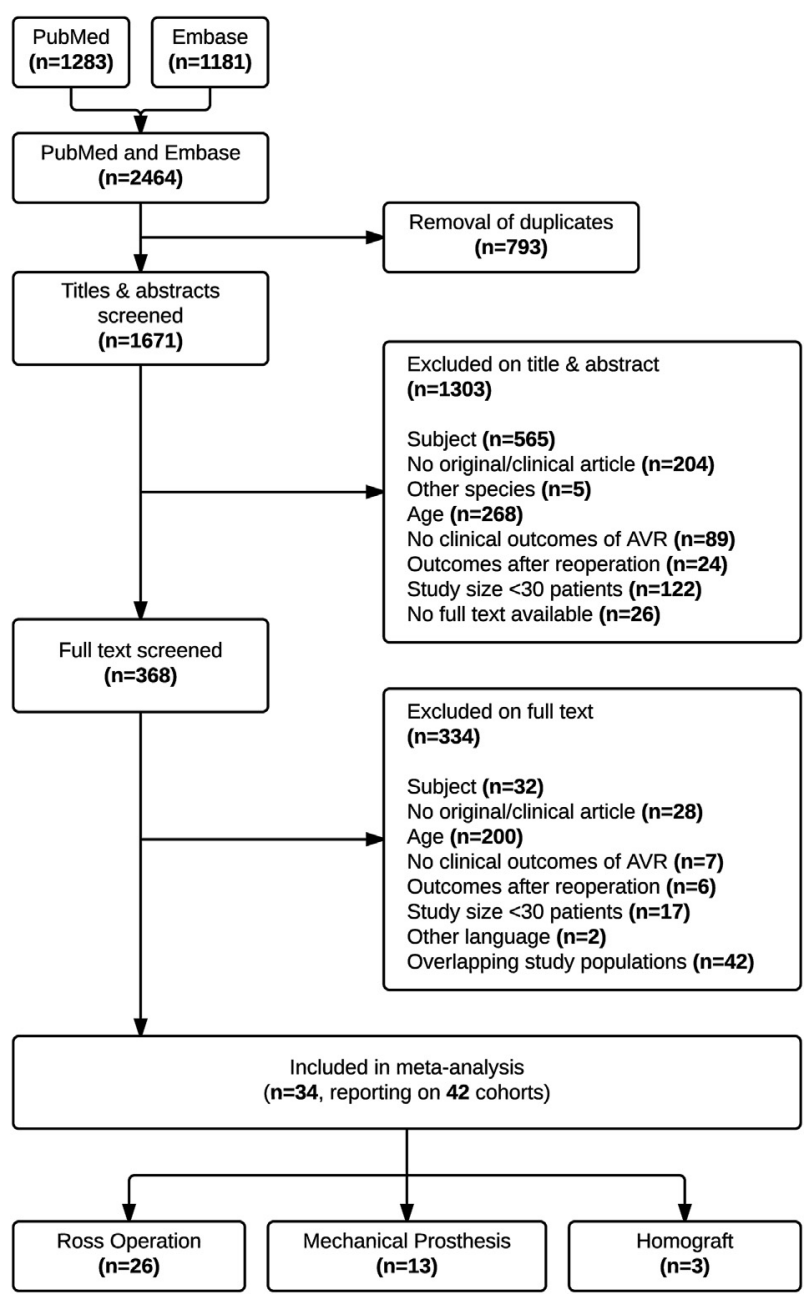

FIGURE 1. Flowchart of study selection.

cardiac interventions $(56.8 \%$ vs $47.6 \%, P=.001)$, whereas the number of concomitant procedures $(27.7 \%$ vs $26.0 \%$, $P=.480)$ and annular enlargement procedures $(19.3 \%$ vs $16.5 \%, P=.127$ ) was comparable between the 2 groups. Paucity of baseline patient data in the HG-AVR group precluded pooled analysis.

\section{Study Outcomes}

Pooled outcome measures (mortality, reoperations, and complications) after the Ross procedure in the general pediatric patient population are shown in Table 2, and for neonates and infants, these are shown in Table 3. Pooled outcome measures after MP-AVR and HG-AVR are shown in Tables 4 and 5, respectively. In studies reporting on postoperative NYHA functional class $(\mathrm{n}=13),{ }^{11,21,28,31,32,34,36,37,41,42,45,47,49} 93 \%$ of the patients were in NYHA class I at last follow-up.

\section{Heterogeneity and Publication Bias}

Significant heterogeneity was found in the pooling of reoperation rates after the Ross procedure and MP-AVR 
TABLE 1. Overview of publications

\begin{tabular}{|c|c|c|c|c|c|c|c|c|c|}
\hline First author & $\begin{array}{c}\begin{array}{c}\text { Year of } \\
\text { publication }\end{array} \\
\end{array}$ & $\begin{array}{c}\text { No. of } \\
\text { patients } \\
\end{array}$ & Study type & $\begin{array}{l}\text { Mean } \\
\text { follow- } \\
\text { up (y) }\end{array}$ & $\begin{array}{r}\text { Mean } \\
\text { age }(y) \\
\end{array}$ & $\begin{array}{c}\text { Cause } \\
\text { (most } \\
\text { frequent) }\end{array}$ & $\begin{array}{c}\text { Previous cardiac } \\
\text { interventions }(\%)^{*}\end{array}$ & $\begin{array}{c}\begin{array}{c}\text { Concomitant } \\
\text { procedures }(\%) \dagger\end{array} \\
\end{array}$ & $\begin{array}{c}\text { Annular } \\
\text { enlargement } \\
\text { procedures (\%)* }\end{array}$ \\
\hline \multicolumn{10}{|l|}{ Ross procedure } \\
\hline Gerosa & 1991 & 43 & Retrospective & 6.9 & 14 & - & 34.9 & 14.0 & - \\
\hline Reddy & 1998 & 41 & Retrospective & 2.6 & $7.8 \ddagger$ & Congenital & 80.5 & 56.1 & 29.3 \\
\hline Elkins & 2001 & 178 & Retrospective & $5.2 \ddagger$ & 9.6 & - & 51.1 & 23.0 & 6.2 \\
\hline Simon & 2001 & 30 & Retrospective & 4.3 & 11.3 & Congenital & - & - & 0.0 \\
\hline Hazekamp & 2005 & 53 & Retrospective & 5.5 & 9.2 & Congenital & 69.8 & 24.5 & 5.7 \\
\hline Bohm & 2006 & 60 & Retrospective & 3.5 & 12.6 & Congenital & 65.0 & 21.7 & 6.7 \\
\hline Kalavrouziotis & 2006 & 35 & Retrospective & 4.1 & 10.6 & - & 85.7 & - & 0.0 \\
\hline Ruzmetov & 2006 & 81 & Retrospective & 5.3 & 5.3 & - & - & - & - \\
\hline Stewart & 2007 & 46 & Retrospective & 5.4 & 12.9 & Congenital & - & 15.2 & 0.0 \\
\hline Kadner & 2008 & 52 & Retrospective & $3.6 \ddagger$ & 5.2 & - & 65.4 & 21.2 & 30.8 \\
\hline Alsoufi & 2009 & 215 & Retrospective & 5.7 & 11.4 & Rheumatic & - & - & 14.9 \\
\hline El Behery & 2009 & 41 & Retrospective & 6 & 10.2 & Congenital & - & 24.4 & - \\
\hline Piccardo & 2009 & 55 & Retrospective & 5.5 & 10 & Congenital & - & 12.7 & 16.4 \\
\hline Charitos & 2012 & 263 & Prospective & 6.9 & 8 & Congenital & 53.6 & - & - \\
\hline Talwar & 2012 & 36 & Retrospective & 7.9 & 11.3 & - & - & 27.8 & - \\
\hline Woods (age < 1 y) & 2012 & 145 & Retrospective & - & $-(<1)$ & - & - & - & - \\
\hline Elder (age < 1 y) & 2013 & 34 & Retrospective & 10.6 & 0.5 & Congenital & - & - & - \\
\hline Khan & 2013 & 68 & Retrospective & 6.7 & $5.9 \ddagger$ & - & - & - & 23.5 \\
\hline Ruzmetov & 2013 & 78 & Retrospective & 8.8 & 11.1 & Congenital & - & 29.5 & 23.1 \\
\hline Tan Tanny & 2013 & 100 & Retrospective & 7.3 & 8.6 & Congenital & - & 29.0 & 29.0 \\
\hline Luciani & 2014 & 305 & Retrospective & 8.4 & 9.4 & - & 30.4 & 29.2 & 23.9 \\
\hline Luciani (age $<1$ y) & 2014 & 37 & Retrospective & 8.4 & 0.3 & - & - & 54.1 & 70.3 \\
\hline Bansal & 2015 & 210 & Retrospective & 5.0 & 8.1 & - & - & 37.1 & - \\
\hline Bansal (age <1 y) & 2015 & 41 & Retrospective & 6.1 & 0.3 & - & - & - & - \\
\hline Nelson & 2015 & 240 & Retrospective & 10.8 & - & Congenital & 73.8 & - & 32.5 \\
\hline Nelson (age < 1 y) & 2015 & 44 & Retrospective & 9.8 & - & Congenital & 75.0 & - & 68.2 \\
\hline Total & & 2409 & & & & & & & \\
\hline \multicolumn{10}{|l|}{ Mechanical prosthesis } \\
\hline Abid & 1992 & 64 & Retrospective & 7 & 12 & Rheumatic & - & 28.1 & 10.9 \\
\hline Cabalka & 1995 & 36 & Retrospective & 3.3 & $-(<18)$ & Congenital & 58.3 & - & - \\
\hline Yamak & 1995 & 37 & Retrospective & 2.9 & 16.6 & Rheumatic & - & 24.3 & 10.8 \\
\hline Champsaur & 1997 & 54 & Retrospective & 5.8 & 12.8 & Congenital & 51.9 & 46.3 & 16.7 \\
\hline Mazzitelli & 1998 & 30 & Retrospective & 6.6 & $-(<18)$ & - & - & - & - \\
\hline Lupinetti & 1999 & 50 & Retrospective & 5.4 & 12.1 & Congenital & 62.0 & 10.0 & 12.0 \\
\hline Alexiou & 2000 & 56 & Retrospective & 7.3 & 11.2 & Congenital & 64.3 & 26.8 & 50.0 \\
\hline Shanmugam & 2005 & 55 & Retrospective & 13 & 13 & Congenital & - & 18.2 & 21.8 \\
\hline Ruzmetov & 2006 & 47 & Retrospective & 7.7 & 7.7 & - & - & - & - \\
\hline Burczynski & 2007 & 55 & Retrospective & 6.4 & 12.8 & Congenital & - & 20.0 & 0.0 \\
\hline Masuda & 2008 & 45 & Retrospective & 9.2 & 9 & - & 44.4 & 33.3 & 55.6 \\
\hline Alsoufi & 2009 & 131 & Retrospective & 8.3 & 14 & Rheumatic & 31.3 & - & 2.3 \\
\hline Khan & 2013 & 36 & Retrospective & 4.6 & $14.0 \ddagger$ & - & - & - & 5.6 \\
\hline Total & & 696 & & & & & & & \\
\hline \multicolumn{10}{|l|}{ Homograft } \\
\hline Gerosa & 1991 & 103 & Retrospective & 8.4 & 12.5 & - & 43.7 & - & - \\
\hline Clarke & 1993 & 47 & Retrospective & 2.3 & 7.1 & - & 89.4 & - & 76.6 \\
\hline Khan & 2013 & 74 & Retrospective & 4 & $4.9 \ddagger$ & - & - & - & 12.2 \\
\hline Total & & 224 & & & & & & & \\
\hline
\end{tabular}

“-" = variable not reported. *Number of patients. †Number of procedures (excluding annular enlargement procedures). $\ddagger$ Median.

and endocarditis rates after the Ross procedure. With regard to reoperations on the aortic valve after the Ross procedure, an outlier in mean follow-up ${ }^{22}$ was identified as an isolated source of heterogeneity. Likewise, an outlier in inclusion period $^{13}$ caused heterogeneity in endocarditis rates after the Ross procedure. Heterogeneity in aortic valve reoperation rates after MP-AVR was also caused by a single outlier, ${ }^{48}$ although we were unable to identify any 
TABLE 2. Pooled outcome estimates after the Ross procedure (excluding studies that concerned only neonates and infants)

\begin{tabular}{|c|c|c|c|c|c|c|c|c|c|}
\hline Study & $\begin{array}{c}\text { Early mortality } \\
\% \\
\end{array}$ & $\begin{array}{c}\text { Late mortality } \\
\% / y\end{array}$ & $\begin{array}{c}\text { SUUD } \\
\% / \mathbf{y}\end{array}$ & $\begin{array}{c}\mathrm{AV} \\
\text { reoperation } \\
\% / \mathbf{y} \\
\end{array}$ & $\begin{array}{c}\text { RVOT } \\
\text { reoperation } \\
\% / y \\
\end{array}$ & $\begin{array}{c}\text { Reoperation for } \\
\text { SVD/NSVD } \\
\% / \mathbf{y} \\
\end{array}$ & $\begin{array}{c}\text { TE/VT } \\
\% / \mathbf{y} \\
\end{array}$ & $\begin{array}{c}\text { Bleeding } \\
\% / y\end{array}$ & $\begin{array}{c}\text { Endocarditis } \\
\% / \mathbf{y} \\
\end{array}$ \\
\hline Gerosa (1991) & $11.63(5.10-26.51)$ & $1.68(0.71-4.01)$ & - & $1.68(0.71-4.01)$ & $0.34(0.05-2.38)$ & $0.34(0.05-2.38)$ & $0.17(0.01-2.69)$ & - & $1.68(0.71-4.01)$ \\
\hline Reddy (1998) & $2.44(0.35-16.90)$ & $0.47(0.03-7.50)$ & $0.47(0.03-7.50)$ & $1.89(0.48-7.45)$ & $0.47(0.03-7.50)$ & $1.89(0.48-7.45)$ & - & - & - \\
\hline Elkins (2001) & $4.49(2.28-8.85)$ & $0.31(0.10-0.95)$ & - & $1.23(0.70-2.16)$ & $0.92(0.48-1.77)$ & $1.95(1.25-3.05)$ & $0.05(0.00-0.82)$ & - & $0.21(0.05-0.82)$ \\
\hline Simon (2001) & $1.67(0.11-26.04)$ & $0.78(0.11-5.46)$ & $0.39(0.02-6.16)$ & $0.78(0.11-5.46)$ & $0.39(0.02-6.16)$ & $0.78(0.11-5.46)$ & $0.39(0.02-6.16)$ & $0.39(0.02-6.16)$ & $0.39(0.02-6.16)$ \\
\hline Hazekamp (2005) & $5.66(1.89-16.99)$ & $1.03(0.33-3.17)$ & $0.17(0.01-2.74)$ & $1.72(0.72-4.09)$ & $1.03(0.33-3.17)$ & $2.74(1.39-5.44)$ & - & - & - \\
\hline Bohm (2006) & $0.83(0.05-13.17)$ & $0.95(0.24-3.78)$ & $0.24(0.01-3.79)$ & $0.24(0.01-3.79)$ & $4.76(2.60-8.72)$ & $4.76(2.60-8.72)$ & $0.24(0.01-3.79)$ & $0.24(0.01-3.79)$ & $0.24(0.01-3.79)$ \\
\hline Kalavrouziotis (2006) & $1.43(0.09-22.39)$ & $0.70(0.10-4.91)$ & $0.35(0.02-5.54)$ & $0.35(0.02-5.54)$ & $1.39(0.35-5.52)$ & $1.39(0.35-5.52)$ & - & - & - \\
\hline Ruzmetov (2006) & $1.23(0.18-8.66)$ & $0.23(0.03-1.65)$ & $0.12(0.01-1.86)$ & $1.63(0.78-3.40)$ & $1.40(0.63-3.09)$ & $3.03(1.77-5.17)$ & $0.12(0.01-1.86)$ & $0.12(0.01-1.86)$ & $0.12(0.01-1.86)$ \\
\hline Stewart (2007) & $1.09(0.07-17.12)$ & $0.20(0.01-3.20)$ & $0.20(0.01-3.20)$ & $2.81(1.35-5.83)$ & $0.40(0.06-2.84)$ & $3.21(1.62-6.35)$ & $0.20(0.01-3.20)$ & $0.20(0.01-3.20)$ & - \\
\hline Kadner (2008) & $9.62(4.18-22.12)$ & $1.61(0.52-4.95)$ & $0.27(0.02-4.27)$ & $1.07(0.27-4.26)$ & $3.22(1.47-7.08)$ & $4.29(2.18-8.46)$ & $0.27(0.02-4.27)$ & - & $0.27(0.02-4.27)$ \\
\hline Alsoufi (2009) & $2.33(0.98-5.53)$ & $0.04(0.00-0.65)$ & $0.04(0.00-0.65)$ & $2.28(1.58-3.29)$ & - & - & $0.04(0.00-0.65)$ & $0.04(0.00-0.65)$ & $0.16(0.04-0.65)$ \\
\hline El Behery (2009) & $4.88(1.26-18.85)$ & $0.20(0.01-3.24)$ & $0.20(0.01-3.24)$ & $0.20(0.01-3.24)$ & $1.63(0.62-4.30)$ & $0.81(0.20-3.23)$ & - & - & $0.81(0.20-3.23)$ \\
\hline Piccardo (2009) & $1.82(0.26-12.68)$ & $0.66(0.17-2.63)$ & $0.17(0.01-2.64)$ & $0.99(0.32-3.06)$ & $0.99(0.32-3.06)$ & $0.66(0.17-2.63)$ & - & - & $0.33(0.05-2.34)$ \\
\hline Charitos (2012) & $3.42(1.80-6.50)$ & $0.58(0.30-1.12)$ & $0.13(0.03-0.52)$ & $0.91(0.54-1.53)$ & $2.72(2.02-3.67)$ & $2.98(2.24-3.96)$ & $0.58(0.30-1.12)$ & $0.03(0.00-0.52)$ & $0.65(0.35-1.20)$ \\
\hline Talwar (2012) & $2.78(0.40-19.19)$ & $1.40(0.53-3.71)$ & $0.18(0.01-2.80)$ & $1.75(0.74-4.18)$ & $0.70(0.18-2.79)$ & - & $0.18(0.01-2.80)$ & $0.18(0.01-2.80)$ & $0.70(0.18-2.79)$ \\
\hline Khan (2013) & $1.47(0.21-10.29)$ & $0.44(0.11-1.75)$ & - & $0.66(0.21-2.03)$ & $3.51(2.17-5.68)$ & - & - & - & - \\
\hline Ruzmetov (2013) & $3.85(1.27-11.67)$ & $0.58(0.22-1.55)$ & $0.29(0.07-1.16)$ & $2.91(1.89-4.49)$ & $2.33(1.44-3.78)$ & $5.24(3.82-7.21)$ & - & - & - \\
\hline Tan Tanny (2013) & $6.00(2.76-13.03)$ & $0.55(0.21-1.46)$ & $0.14(0.02-0.97)$ & $1.23(0.64-2.36)$ & $2.74(1.78-4.22)$ & - & - & - & - \\
\hline Luciani (2014) & $3.28(1.78-6.03)$ & $0.47(0.27-0.82)$ & $0.02(0.00-0.31)$ & $1.44(1.05-1.99)$ & $1.44(1.05-1.99)$ & $2.77(2.20-3.49)$ & $0.08(0.02-0.31)$ & $0.02(0.00-0.31)$ & $0.08(0.02-0.31)$ \\
\hline Bansal (2015) & $4.29(2.26-8.12)$ & $0.05(0.00-0.76)$ & $0.05(0.00-0.76)$ & - & - & - & - & - & - \\
\hline Nelson (2015) & $4.17(2.27-7.64)$ & $0.66(0.41-1.05)$ & - & $2.66(2.11-3.36)$ & $2.20(1.70-2.84)$ & - & - & - & - \\
\hline Pooled (random effects) & $4.20(3.37-5.22)$ & $0.64(0.49-0.84)$ & $0.16(0.09-0.29)$ & $1.60(1.27-2.02)$ & $1.91(1.50-2.44)$ & $2.75(2.13-3.53)$ & $0.22(0.11-0.43)$ & $0.10(0.04-0.27)$ & $0.40(0.22-0.73)$ \\
\hline Heterogeneity test & $\begin{array}{c}\chi^{2} 20.78(P=.41) \\
\mathrm{I}^{2}=3 \%\end{array}$ & $\begin{array}{c}\chi^{2} 23.77(P=.25) \\
I^{2}=16 \%\end{array}$ & $\begin{array}{c}\chi^{2} 6.32(P=.98) \\
\mathrm{I}^{2}=0 \%\end{array}$ & $\begin{array}{c}\chi^{2} 40.97(P=.00) \\
I^{2}=54 \%\end{array}$ & $\begin{array}{c}\chi^{2} 44.49(P=.00) \\
I^{2}=59 \%\end{array}$ & $\begin{array}{c}\chi^{2} 35.59(P=.00) \\
\mathrm{I}^{2}=60 \%\end{array}$ & $\begin{array}{c}\chi^{2} 12.09(P=.27) \\
\mathrm{I}^{2}=17 \%\end{array}$ & $\begin{array}{c}\chi^{2} 4.08(P=.77) \\
\mathrm{I}^{2}=0 \%\end{array}$ & $\begin{array}{c}\chi^{2} 21.60(P=.03) \\
\mathrm{I}^{2}=49 \%\end{array}$ \\
\hline
\end{tabular}

death; $A V$, aortic valve; $R V O T$, right ventricular outflow tract; $S V D$, structural valv 
TABLE 3. Pooled outcome estimates after the Ross procedure in neonates and infants

\begin{tabular}{|c|c|c|c|c|c|c|c|c|c|}
\hline Study & $\begin{array}{c}\text { Early mortality } \\
\% \\
\end{array}$ & $\begin{array}{c}\text { Late mortality } \\
\% / \mathbf{y}\end{array}$ & $\begin{array}{l}\text { SUUD } \\
\% / \mathbf{y}\end{array}$ & $\begin{array}{c}\mathrm{AV} \\
\text { reoperation } \\
\% / \mathbf{y}\end{array}$ & $\begin{array}{c}\text { RVOT } \\
\text { reoperation } \\
\% / \mathbf{y} \\
\end{array}$ & $\begin{array}{c}\text { Reoperation } \\
\text { for SVD/ } \\
\text { NSVD } \\
\% / y \\
\end{array}$ & $\begin{array}{c}\text { TE/VT } \\
\% / \mathbf{y}\end{array}$ & $\begin{array}{c}\text { Bleeding } \\
\% / \mathbf{y}\end{array}$ & $\begin{array}{c}\text { Endocarditis } \\
\% / \mathbf{y}\end{array}$ \\
\hline Woods (2012) & $15.86(10.90-23.08)$ & - & - & - & - & - & - & - & - \\
\hline Elder (2013) & $11.76(4.69-29.54)$ & $0.14(0.01-2.21)$ & $0.14(0.01-2.21)$ & $0.28(0.04-1.96)$ & $4.16(2.54-6.83)$ & - & - & - & $0.28(0.04-1.96)$ \\
\hline Luciani (2014) & $21.62(11.71-39.93)$ & $2.57(1.30-5.10)$ & - & $1.93(0.87-4.26)$ & - & - & - & - & - \\
\hline Bansal (2015) & $17.07(8.70-33.52)$ & $0.20(0.01-3.19)$ & $0.20(0.01-3.19)$ & - & - & - & - & - & - \\
\hline Nelson (2015) & $18.18(9.71-34.03)$ & $0.70(0.23-2.15)$ & - & $0.46(0.12-1.85)$ & $4.41(2.84-6.84)$ & - & - & - & - \\
\hline Pooled & $16.88(13.12-21.73)^{*}$ & $0.76(0.21-2.78)^{*}$ & $0.17(0.02-1.18) \dagger$ & $1.14(0.60-2.18) \dagger$ & $4.30(3.09-5.97) \dagger$ & - & - & - & $0.28(0.04-1.96) \dagger$ \\
\hline Heterogeneity test & $\begin{array}{c}\chi^{2} 1.38(P=.85) \\
\mathrm{I}^{2}=0 \%\end{array}$ & $\begin{array}{c}\chi^{2} 9.05(P=.03) \\
I^{2}=67 \%\end{array}$ & $\begin{array}{c}\chi^{2} 0.03(P=.85) \\
\mathrm{I}^{2}=0 \%\end{array}$ & $\begin{array}{c}\chi^{2} 5.32(P=.07) \\
I^{2}=62 \%\end{array}$ & $\begin{array}{c}\chi^{2} 0.03(P=.87) \\
\mathrm{I}^{2}=0 \%\end{array}$ & $\begin{array}{l}- \\
-\end{array}$ & $\begin{array}{l}- \\
-\end{array}$ & $\begin{array}{l}- \\
-\end{array}$ & - \\
\hline
\end{tabular}

explanatory study or patient characteristics. Exploratory exclusion of these studies eliminated significant heterogeneity but did not cause a major change in the pooled estimates.

We were unable to identify the cause of heterogeneity in RVOT reoperations after the Ross procedure. The funnel plots showed evidence of possible publication bias in all outcome measures (Figure E1). Smaller studies with relatively high event rate estimates seemed less likely to be published.

\section{DISCUSSION}

This is the first study that systematically compiles the available evidence on reported outcome after contemporary AVR in the pediatric population into a comprehensive overview. It highlights the imperfections of currently available aortic valve substitutes in children. It seems that the Ross procedure in children aged more than 1 year is associated with low early and late mortality rates, lower compared with MP-AVR and HG-AVR. However, this study also shows that RVOT reoperations after the Ross procedure are required in approximately $20 \%$ of the general pediatric patient population and in more than $40 \%$ of neonates and infants in the first postoperative decade. In addition, the results of our study indicate that HG-AVR is associated with a significantly higher rate of aortic valve reoperations compared with both the MP-AVR and the Ross procedure. The initial search resulted in only 6 studies on bioprostheses, all of which had a sample size of less than 20 patients, reflecting the abandonment of their use in pediatric AVR in contemporary practice as a result of their high rates of early degeneration, calcification, and structural failure in young recipients. $8,9,15-17,23,45$

\section{Early Mortality}

The observed differences in early mortality risk between the different heart valve substitutes are probably mainly driven by patient-related factors, such as patient age,

TABLE 4. Pooled outcome estimates after aortic valve replacement with mechanical prosthesis

\begin{tabular}{|c|c|c|c|c|c|c|c|c|}
\hline Study & $\begin{array}{c}\text { Early mortality } \\
\%\end{array}$ & $\begin{array}{c}\text { Late mortality } \\
\% / \mathbf{y}\end{array}$ & $\begin{array}{l}\text { SUUD } \\
\% / \mathbf{y}\end{array}$ & $\begin{array}{c}\text { AV reoperation } \\
\% / y\end{array}$ & $\begin{array}{c}\text { Reoperation } \\
\text { for SVD/NSVD } \\
\% / y\end{array}$ & $\begin{array}{l}\text { TE/VT } \\
\% / \mathbf{y}\end{array}$ & $\begin{array}{c}\text { Bleeding } \\
\% / \mathbf{y}\end{array}$ & $\begin{array}{c}\text { Endocarditis } \\
\% / \mathbf{y}\end{array}$ \\
\hline Abid (1992) & $12.50(6.54-23.90)$ & $2.60(1.41-4.79)$ & $0.13(0.01-2.07)$ & $1.30(0.54-3.10)$ & $0.78(0.25-2.41)$ & $1.30(0.54-3.10)$ & $0.52(0.13-2.07)$ & $0.78(0.25-2.41)$ \\
\hline Cabalka (1995) & $5.56(1.44-21.36)$ & $0.85(0.12-6.02)$ & - & $0.43(0.03-6.79)$ & $0.43(0.03-6.79)$ & $0.85(0.12-6.02)$ & $1.71(0.43-6.75)$ & $0.43(0.03-6.79)$ \\
\hline Yamak (1995) & $8.11(2.74-23.99)$ & $0.49(0.03-7.83)$ & $0.49(0.03-7.83)$ & $0.49(0.03-7.83)$ & $0.49(0.03-7.83)$ & $0.49(0.03-7.83)$ & $0.49(0.03-7.83)$ & $0.49(0.03-7.83)$ \\
\hline Champsaur (1997) & $12.96(6.49-25.87)$ & $2.30(1.04-5.07)$ & $0.19(0.01-3.05)$ & $1.15(0.37-3.54)$ & $1.15(0.37-3.54)$ & $0.77(0.19-3.05)$ & $0.38(0.05-2.71)$ & - \\
\hline Mazzitelli (1998) & $1.67(0.11-26.04)$ & $1.52(0.49-4.66)$ & $0.25(0.02-4.02)$ & $2.53(1.06-6.00)$ & $1.52(0.49-4.66)$ & $1.01(0.25-4.01)$ & $0.51(0.07-3.57)$ & - \\
\hline Lupinetti (1999) & $10.00(4.35-22.97)$ & $1.48(0.56-3.91)$ & $0.18(0.01-2.94)$ & $3.69(2.01-6.78)$ & $2.58(1.24-5.37)$ & $1.11(0.36-3.41)$ & $0.18(0.01-2.94)$ & $0.74(0.19-2.94)$ \\
\hline Alexiou (2000) & $5.36(1.78-16.11)$ & $0.74(0.24-2.29)$ & $0.12(0.01-1.97)$ & $0.74(0.24-2.29)$ & $0.49(0.12-1.97)$ & $0.49(0.12-1.97)$ & $0.12(0.01-1.97)$ & $0.12(0.01-1.97)$ \\
\hline Shanmugam (2005) & $0.91(0.06-14.35)$ & $0.15(0.02-1.07)$ & $0.08(0.00-1.20)$ & $0.60(0.23-1.60)$ & $0.45(0.15-1.40)$ & $0.08(0.00-1.20)$ & $0.15(0.02-1.07)$ & $0.30(0.08-1.20)$ \\
\hline Ruzmetov (2006) & $6.38(2.14-19.08)$ & $0.83(0.27-2.56)$ & $0.14(0.01-2.20)$ & $1.38(0.58-3.30)$ & $0.83(0.27-2.56)$ & $0.14(0.01-2.20)$ & $0.14(0.01-2.20)$ & $0.55(0.14-2.20)$ \\
\hline Burczynski (2007) & $0.91(0.06-14.35)$ & $0.57(0.14-2.26)$ & $0.14(0.01-2.26)$ & $0.28(0.04-2.01)$ & $0.14(0.01-2.26)$ & $0.57(0.14-2.26)$ & $0.14(0.01-2.26)$ & $0.57(0.14-2.26)$ \\
\hline Masuda (2008) & $2.22(0.32-15.43)$ & $0.48(0.12-1.93)$ & $0.24(0.03-1.71)$ & $0.48(0.12-1.93)$ & $0.24(0.03-1.71)$ & $0.97(0.36-2.56)$ & - & $0.24(0.03-1.71)$ \\
\hline Alsoufi (2009) & $6.11(3.12-11.95)$ & $1.75(1.12-2.73)$ & - & $0.74(0.37-1.47)$ & - & $0.37(0.14-0.98)$ & $0.28(0.09-0.85)$ & $0.09(0.01-0.65)$ \\
\hline Khan (2013) & $1.39(0.09-21.78)$ & $0.60(0.09-4.26)$ & - & $0.60(0.09-4.26)$ & - & $1.21(0.30-4.79)$ & - & - \\
\hline Pooled (random effects) & $7.34(5.21-10.34)$ & $1.23(0.85-1.79)$ & $0.18(0.08-0.41)$ & 1.07 (0.68-1.68) & $0.86(0.53-1.42)$ & $0.76(0.53-1.09)$ & $0.39(0.22-0.68)$ & $0.45(0.27-0.75)$ \\
\hline Heterogeneity test & $\begin{array}{c}\chi^{2} 14.78(P=.32) \\
\mathrm{I}^{2}=18 \%\end{array}$ & $\begin{array}{c}\chi^{2} 18.79(P=.13) \\
\mathrm{I}^{2}=36 \%\end{array}$ & $\begin{array}{c}\chi^{2} 1.22(P=1.00) \\
\mathrm{I}^{2}=0 \%\end{array}$ & $\begin{array}{c}\chi^{2} 25.57(P=.01) \\
\mathrm{I}^{2}=53 \%\end{array}$ & $\begin{array}{c}\chi^{2} 14.81(P=.19) \\
\mathrm{I}^{2}=32 \%\end{array}$ & $\begin{array}{c}\chi^{2} 9.62(P=.72) \\
\mathrm{I}^{2}=0 \%\end{array}$ & $\begin{array}{c}\chi^{2} 7.97(P=.72) \\
\mathrm{I}^{2}=0 \%\end{array}$ & $\begin{array}{c}\chi^{2} 5.67(P=.84) \\
\mathrm{I}^{2}=0 \%\end{array}$ \\
\hline
\end{tabular}

Data expressed as percentage (95\% CI). "_" = variable not reported. In case an event was reported not to occur, for pooling purposes it was assumed that 0.5 patient experienced the event. SUUD, Sudden, unexpected, unexplained death; $A V$, aortic valve; $S V D$, structural valve deterioration; $N S V D$, nonstructural valve dysfunction; $T E$, thromboembolism; $V T$, valve thrombosis. 
TABLE 5. Pooled outcome estimates after aortic valve replacement with homograft

\begin{tabular}{|c|c|c|c|c|c|c|c|c|}
\hline Study & $\begin{array}{c}\text { Early mortality } \\
\%\end{array}$ & $\begin{array}{c}\text { Late mortality } \\
\% / \mathbf{y}\end{array}$ & $\begin{array}{c}\text { SUUD } \\
\% / \mathbf{y}\end{array}$ & $\begin{array}{c}\text { AV reoperation } \\
\% / \mathbf{y}\end{array}$ & $\begin{array}{c}\text { Reoperation } \\
\text { for SVD/NSVD } \\
\% / \mathbf{y}\end{array}$ & $\begin{array}{c}\text { TE/VT } \\
\% / \mathbf{y}\end{array}$ & $\begin{array}{c}\text { Bleeding } \\
\% / y\end{array}$ & $\begin{array}{c}\text { Endocarditis } \\
\% / \mathbf{y}\end{array}$ \\
\hline Gerosa (1991) & $15.53(9.90-24.37)$ & $1.62(0.96-2.72)$ & - & $2.77(1.87-4.12)$ & $2.43(1.59-3.70)$ & $0.06(0.00-0.92)$ & $0.06(0.00-0.92)$ & $0.23(0.06-0.92)$ \\
\hline Clarke (1993) & $12.77(6.05-26.95)$ & $0.93(0.13-6.51)$ & $0.46(0.03-7.35)$ & $6.48(3.16-13.26)$ & $5.55(2.55-12.08)$ & $0.93(0.13-6.51)$ & $2.78(0.91-8.47)$ & $1.85(0.47-7.30)$ \\
\hline Khan (2013) & $4.05(1.34-12.28)$ & $1.69(0.71-4.03)$ & - & $9.12(6.37-13.07)$ & - & - & - & - \\
\hline Pooled (fixed effects) & $12.82(8.91-18.46)$ & $1.59(1.03-2.46)$ & $0.46(0.03-7.35)$ & $5.44(4.24-6.98)$ & $2.93(2.02-4.25)$ & $0.37(0.07-1.82)$ & $1.62(0.57-4.55)$ & $0.66(0.25-1.75)$ \\
\hline Heterogeneity test & $\begin{array}{c}\chi^{2} 8.37(P=.04) \\
\mathrm{I}^{2}=76 \%\end{array}$ & $\begin{array}{c}\chi^{2} 0.51(P=.91) \\
\mathrm{I}^{2}=0 \%\end{array}$ & - & $\begin{array}{c}\chi^{2} 13.89(P=.00) \\
\mathrm{I}^{2}=86 \%\end{array}$ & $\begin{array}{c}\chi^{2} 0.88(P=.64) \\
\mathrm{I}^{2}=0 \%\end{array}$ & $\begin{array}{c}\chi^{2} 2.95(P=.23) \\
\mathrm{I}^{2}=66 \%\end{array}$ & $\begin{array}{c}\chi^{2} 3.14(P=.21) \\
\mathrm{I}^{2}=68 \%\end{array}$ & $\begin{array}{c}\chi^{2} 1.54(P=.46) \\
\mathrm{I}^{2}=35 \%\end{array}$ \\
\hline
\end{tabular}

Data expressed as percentage $(95 \% \mathrm{CI})$. "-" = variable not reported. In case an event was reported not to occur, for pooling purposes it was assumed that 0.5 patient experienced the event. SUUD, Sudden, unexpected, unexplained death; $A V$, aortic valve; $S V D$, structural valve deterioration; $N S V D$, nonstructural valve dysfunction; $T E$, thromboembolism; $V T$, valve thrombosis.

urgency of the procedure, preoperative hemodynamic status, and disease cause.

Differences in patient characteristics, surgical technique, and additional procedures performed at the time of MP-AVR may explain the higher early mortality rate in this patient group. However, our pooled analysis of patient characteristics and surgical details revealed differences that were not consistently in favor of the Ross procedure. Whereas rheumatic valve disease was more frequent in patients undergoing MP-AVR, patients undergoing the Ross procedure more frequently had undergone previous cardiac interventions. The number of concomitant procedures and annular enlargement procedures was comparable between the 2 groups. The 2 included studies that compared the characteristics of patients undergoing the Ross procedure with those of mechanical valve recipients further support the differences we found in pooled baseline patient characteristics. $^{23,31}$ Although we did not find conclusive evidence of selection bias, residual hidden selection bias may explain the higher mortality rate associated with MP-AVR. With regard to the Ross procedure, younger age at the time of operation seems to be associated with a less favorable outcome and is most likely representative of very complex (critical) aortic stenosis. This is confirmed by our observations in the studies concerning the Ross procedure in neonates and infants that showed a substantially higher early mortality risk than the older pediatric patients undergoing the Ross. Next to patientrelated factors, the era of operation may have influenced early mortality risk, analogous to findings in young adults who undergo valve replacement. ${ }^{54}$ The early mortality risks were higher in studies conducted in the early 1990s when compared with more recent studies. ${ }^{13,49,50,53}$ Improved early patient outcome in more recent years is most likely the result of improvements in diagnostic workup, surgical timing, intensive care, and anesthesia.

\section{Late Mortality}

Similar to observations in young adult patient populations, ${ }^{55}$ the Ross procedure in children is associated with significantly lower late mortality rates compared with
MP-AVR and HG-AVR (0.64\%/y vs $1.23 \%$ /y vs $1.59 \% / y$, respectively). PPM and suboptimal hemodynamic performance of MPs and HGs may have contributed to the observed excess mortality, particularly in growing children.

In addition, as with early mortality, the higher late mortality rate in MP-AVR may be explained by the fact that children who undergo MP-AVR more often have rheumatic valve disease and connective tissue disease, which may have given rise to substantial selection bias, although we did not find conclusive evidence thereof. ${ }^{31}$

In contrast to the high early mortality risk of the Ross procedure in neonates and infants, late mortality rates in these patients are low and comparable to late mortality in the older pediatric patients undergoing the Ross procedure.

As observed with early mortality, late mortality rates after MP-AVR seem to be dependent on the era of operation, with improved survival in patients undergoing operation more recently. The only exception to this time-dependent improved survival was the study published by Alsoufi and colleagues. ${ }^{31}$ This study describes a population of children with predominantly rheumatic valves in a developing country with suboptimal anticoagulation compliance, which might explain this observation. Late survival after the Ross procedure seems to leave little room for improvement in more recent years.

\section{Reoperations}

The rate of reoperations on the aortic valve was comparable for the Ross procedure and MP-AVR, although the indication for aortic valve reoperation differs. Although patients with an MP tend to outgrow the prosthetic valve, patients undergoing the Ross procedure often experience valve insufficiency caused by dilatation of the neoaortic root. Compared with both the Ross procedure and MP-AVR, HG-AVR was associated with a significantly higher aortic valve reoperation rate, due to both PPM in growing children and early degeneration. ${ }^{56,57}$

One of the major disadvantages of the Ross procedure is that single valve disease is treated with double valve replacement, placing both valves at risk of degeneration and reoperation. Failure of the pulmonary valve substitute, 
although usually less life threatening, does pose an additional risk of reoperation or percutaneous reintervention. When the additional risk of reoperations on the RVOT associated with the Ross procedure is taken into account, the Ross procedure is associated with a higher total reoperation rate compared with MP-AVR in the first postoperative decade, with a further increase in reoperation rates to be expected in the second postoperative decade. ${ }^{6}$ This aspect of the Ross procedure needs to be addressed clearly to (parents of) children who are facing AVR. Of note, for neonates and infants undergoing the Ross procedure, aortic valve reoperation rates seem to be lower, whereas RVOT reoperation rates are 2 times higher compared with older children after the Ross procedure. The latter can be explained by the rapid child growth at a younger age. Studies on outcome after reoperative AVR in children and young adults report early and late mortality rates comparable to those we observed after primary AVR, which suggests that reoperations, although challenging, are safe. $^{27,58-61}$

\section{Valve-Related Complications}

When evaluating valve-related event occurrence after adult AVR with mechanical versus biological valve substitutes, basically the burden of anticoagulation therapy is compared with the burden of reoperation. In children, the growth-dependent increase in PPM needs to be considered. The present study confirms that the Ross procedure is associated with significantly lower TE/VT rates compared with MP-AVR $(0.22 \% / y ; 95 \%$ confidence interval [CI], $0.11-0.43 / \mathrm{y}$ vs $0.76 \% / \mathrm{y} ; 95 \% \mathrm{CI}, 0.53-1.09 / \mathrm{y}$, respectively), and there was a trend toward lower bleeding rates $(0.10 \% / \mathrm{y} ; 95 \%$ CI, $0.04-0.27 / \mathrm{y}$ vs $0.39 \% / \mathrm{y} ; 95 \%$ CI, $0.22-0.68 / y$, respectively). Pooled SVD and NSVD rates after the Ross procedure are substantially higher compared with MP-AVR.

Of note, the only prospective study in this systematic review reported a very high TE/VT event rate of $0.58 \% / y$ for patients undergoing the Ross ${ }^{27}$ compared with the other retrospective Ross studies. The prospective design of this study is the most likely explanation for this observation because there will have been less recall bias compared with retrospective studies.

As expected, pediatric HG-AVR is associated with higher valve deterioration rates than both the Ross procedure and MP-AVR. This is in line with earlier reports that show accelerated calcification and degeneration of the HG in the aortic position in children. ${ }^{57}$

With regard to pooled endocarditis rates, the Ross procedure and MP-AVR were comparable, whereas HG-AVR was associated with a higher rate of endocarditis. This may be explained by the fact that this finding is based on a single study in which a relatively large proportion of patients $(89 \%)$ had undergone at least 1 previous cardiac intervention, which is known to be associated with prosthetic valve endocarditis. ${ }^{62}$

\section{Functional Health Status}

The majority of studies that reported NYHA functional class reported a large proportion of patients being in NYHA class I at last follow-up. However, the NYHA classification has not been designed for children, which most likely explains why preoperative NYHA classification was not assessed in most studies. Therefore, it is not possible to determine improvement in functional performance after AVR. To determine the effect of AVR on functional performance, both preoperative and postoperative functional classification should be registered for every patient. Also, questionnaires are needed to provide more insight in quality of life in relation to heart valve prostheses. There is some evidence for better quality of life after the Ross procedure than after MP-AVR in young adult patients, but more studies are needed. ${ }^{63}$

\section{Source of Heterogeneity}

Although heterogeneity was considerable in our meta-analysis and may have led to inaccurate results, we pursued a thorough examination of possible sources of heterogeneity. The year of operation, ranging from 1964 to 2013 among the included studies, may have affected the results because evolution of operative techniques seems to have led to lower operative risk. Furthermore, the heterogeneity in RVOT reoperation rates after the Ross procedure may be explained by possible interinstitutional variation in indications for RVOT reoperation. The cause of the higher aortic valve reoperation rate reported by Lupinetti and colleagues ${ }^{48}$ in comparison with the other included studies remains to be elucidated.

Our findings of largely unchanged pooled estimates after exploratory exclusion of the identified sources of heterogeneity suggest that the heterogeneity caused by aforementioned studies did not have a substantial impact on the pooled estimates of the outcomes discussed and, thus, did not compromise the validity of these estimates.

\section{Future Perspectives}

Recent clinical practice guidelines for adult valvular heart disease recommend the Ross procedure to be reserved for patients in whom anticoagulation is contraindicated. ${ }^{64}$ Our results show that in particular in younger growing children the Ross procedure may be beneficial in a larger group of patients when performed in centers of expertise. Although the included studies provide limited insight into the impact of patient characteristics on outcome with the different valve options, the Ross procedure seems to be associated with more favorable early and late survival in the first postoperative decade. However, the Ross procedure is also associated with a substantial reoperation rate in 
the first postoperative decade, and a further increase in reoperation rates is to be expected in the second postoperative decade. ${ }^{6}$ Unfortunately, no firm conclusions can be drawn for individual patients because most of the included publications have a mixed study population with regard to patient characteristics and cause of valve disease.

When repair of the aortic valve is not possible or has failed, replacement may be unavoidable. In search of the best AVR procedure in children, heart valve tissue engineering is a promising development. ${ }^{65-67}$ Tissueengineered valve technology is still in its infancy but may be a solution in the future with the prospect of a durable living heart valve that adapts to the growing child. This innovative technology aims at avoiding reoperations and improving long-term outcome after AVR and hopefully will provide a more durable solution for children requiring AVR.

\section{Study Limitations}

The present study is a systematic review and metaanalysis of observational studies, all but 1 retrospective in design. As such, the inherent limitations of meta-analyses and combining data from retrospective observational studies should be taken into consideration. ${ }^{68}$ Selection bias may have affected the observed outcomes because unpublished data, abstracts, and presentations were not included. Finally, the mean follow-up of the included studies does not allow extensions of the observed outcomes beyond the first postoperative decade.

\section{CONCLUSIONS}

Despite improvements in diagnostic workup, surgical timing, and expertise in pediatric aortic valve repair techniques, AVR may be unavoidable. Results of AVR in children remain suboptimal with the currently available valve substitutes. The present study illustrates that both the Ross procedure and MP-AVR, although the most commonly used procedures for AVR in children, are associated with suboptimal outcome, reflecting the urgent need for reliable and durable repair techniques and innovative replacement solutions for this challenging group of patients.

\section{Conflict of Interest Statement}

Authors have nothing to disclose with regard to commercial support.

\section{References}

1. Akins CW, Miller DC, Turina MI, Kouchoukos NT, Blackstone EH, Grunkemeier GL, et al. Guidelines for reporting mortality and morbidity after cardiac valve interventions. Ann Thorac Surg. 2008;85:1490-5.

2. Elkins R. The Ross operation: applications to children. Semin Thorac Cardiovasc Surg. 1996;8:345-9.

3. da Costa FD, Pereira EW, Barboza LE, Haggi Filho H, Collatusso C, Gomes CH, et al. Ten-year experience with the Ross operation. Arq Bras Cardiol. 2006;87:583-91.
4. Elkins RC, Thompson DM, Lane MM, Elkins CC, Peyton MD. Ross operation: 16-year experience. J Thorac Cardiovasc Surg. 2008;136:623-30. 630.e1-5.

5. Takkenberg JJ, Kappetein AP, van Herwerden LA, Witsenburg M Van Osch-Gevers L, Bogers AJ. Pediatric autograft aortic root replacement: a prospective follow-up study. Ann Thorac Surg. 2005;80:1628-33.

6. Mokhles MM, Rizopoulos D, Andrinopoulou ER, Bekkers JA, Roos-Hesselink JW, Lesaffre E, et al. Autograft and pulmonary allograft performance in the second post-operative decade after the Ross procedure: insights from the Rotterdam Prospective Cohort Study. Eur Heart J. 2012;33:2213-24.

7. Brown JW, Ruzmetov M, Vijay P, Rodefeld MD, Turrentine MW. Surgery for aortic stenosis in children: a 40-year experience. Ann Thorac Surg. 2003;76:1398-411.

8. Karamlou T, Jang K, Williams WG, Caldarone CA, Van Arsdell G, Coles JG, et al. Outcomes and associated risk factors for aortic valve replacement in 160 children: a competing-risks analysis. Circulation. 2005;112:3462-9.

9. Turrentine MW, Ruzmetov M, Vijay P, Bills RG, Brown JW. Biological versus mechanical aortic valve replacement in children. Ann Thorac Surg. 2001;71: S356-60.

10. Starnes VA, Luciani GB, Wells WJ, Allen RB, Lewis AB. Aortic roo replacement with the pulmonary autograft in children with complex left heart obstruction. Ann Thorac Surg. 1996;62:442-9.

11. Elkins RC, Lane MM, McCue C. Ross operation in children: late results. J Heart Valve Dis. 2001;10:736-41.

12. van Hagen IM, Roos-Hesselink JW, Ruys TP, Merz WM, Goland S, Gabriel H, et al. Pregnancy in Women With a Mechanical Heart Valve: Data of the European Society of Cardiology Registry of Pregnancy and Cardiac Disease (ROPAC). Circulation. 2015;132:132-42.

13. Gerosa G, McKay R, Davies J, Ross DN. Comparison of the aortic homograft and the pulmonary autograft for aortic valve or root replacement in children. J Thorac Cardiovasc Surg. 1991;102:51-61.

14. Takkenberg JJ, Klieverik LM, Bekkers JA, Kappetein AP, Roos JW Eijkemans MJ, et al. Allografts for aortic valve or root replacement: insights from an 18-year single-center prospective follow-up study. Eur J Cardiothorac Surg. 2007:31:851-9.

15. Mazzitelli D, Guenther T, Schreiber C, Wottke M, Michel J, Meisner H. Aortic valve replacement in children: are we on the right track? Eur J Cardiothorac Surg. 1998;13:565-71.

16. Ruzmetov M, Vijay P, Rodefeld MD, Turrentine MW, Brown JW. Evolution of aortic valve replacement in children: a single center experience. Int J Cardiol. 2006:113:194-200.

17. Wada J, Yokoyama M, Hashimoto A, Imai Y, Kitamura N, Takao A, et al Long-term follow-up of artificial valves in patients under 15 years old. Ann Thorac Surg. 1980;29:519-21.

18. Ali A, Lim E, Halstead J, Ashrafian H, Ali Z, Khalpey Z, et al. Porcine or human stentless valves for aortic valve replacement? Results of a 10-year comparative study. J Heart Valve Dis. 2003;12:430-5.

19. Moher D, Liberati A, Tetzlaff J, Altman DG, Group P. Preferred reporting items for systematic reviews and meta-analyses: the PRISMA statement. PLoS Med. 2009;6:e1000097.

20. Akins CW, Miller DC, Turina MI, Kouchoukos NT, Blackstone EH Grunkemeier GL, et al. Guidelines for reporting mortality and morbidity after cardiac valve interventions. J Thorac Cardiovasc Surg. 2008;135:732-8.

21. Tan Tanny SP, Yong MS, d'Udekem Y, Kowalski R, Wheaton G, D'Orsogna L, et al. Ross procedure in children: 17-year experience at a single institution. J Am Heart Assoc. 2013;2:e00153.

22. Ruzmetov M, Geiss DM, Shah JJ, Buckley K, Fortuna RS. The Ross-Konno is a high-risk procedure when compared with the Ross operation in children. Ann Thorac Surg. 2013;95:670-5.

23. Khan MS, Samayoa AX, Chen DW, Petit CJ, Fraser CD Jr. Contemporary experience with surgical treatment of aortic valve disease in children. $J$ Thorac Cardiovasc Surg. 2013;146:512-20.

24. Elder RW, Quaegebeur JM, Bacha EA, Chen JM, Bourlon F, Williams IA. Outcomes of the infant Ross procedure for congenital aortic stenosis followed into adolescence. J Thorac Cardiovasc Surg. 2013;145:1504-11.

25. Woods RK, Pasquali SK, Jacobs ML, Austin EH, Jacobs JP, Krolikowski M, et al Aortic valve replacement in neonates and infants: an analysis of the Society of Thoracic Surgeons Congenital Heart Surgery Database. J Thorac Cardiovasc Surg. 2012;144:1084-9.

26. Talwar S, Malankar D, Garg S, Choudhary SK, Saxena A, Velayoudham D, et al Aortic valve replacement with biological substitutes in children. Asian Cardiovasc Thorac Ann. 2012;20:518-24. 
27. Charitos EI, Takkenberg JJ, Hanke T, Gorski A, Botha C, Franke U, et al. Reoperations on the pulmonary autograft and pulmonary homograft after the Ross procedure: An update on the German Dutch Ross Registry. J Thorac Cardiovasc Surg. 2012;144:813-23.

28. Shinkawa T, Bove EL, Hirsch JC, Devaney EJ, Ohye RG. Intermediate-term results of the Ross procedure in neonates and infants. Ann Thorac Surg. 2010; 89:1827-32.

29. Frigiola A, Varrica A, Satriano A, Giamberti A, Pome G, Abella R, et al. Neoaortic valve and root complex evolution after Ross operation in infants, children, and adolescents. Ann Thorac Surg. 2010;90:1278-85.

30. El Behery S, Rubay J, Sluysmans T, Absil B, Ovaert C. Midterm results of the Ross procedure in a pediatric population: bicuspid aortic valve is not a contraindication. Pediatr Cardiol. 2009;30:219-24.

31. Alsoufi B, Al-Halees Z, Manlhiot C, McCrindle BW, Al-Ahmadi M, Sallehuddin A, et al. Mechanical valves versus the Ross procedure for aortic valve replacement in children: propensity-adjusted comparison of long-term outcomes. J Thorac Cardiovasc Surg. 2009;137:362-70.e9.

32. Kadner A, Raisky O, Degandt A, Tamisier D, Bonnet D, Sidi D, et al. The Ross procedure in infants and young children. Ann Thorac Surg. 2008;85:803-8.

33. Stewart RD, Backer CL, Hillman ND, Lundt C, Mavroudis C. The Ross operation in children: effects of aortic annuloplasty. Ann Thorac Surg. 2007; 84:1326-30.

34. Kalavrouziotis G, Raja S, Ciotti G, Karunaratne A, Corno AF, Pozzi M. Medium-term results from pulmonary autografts after the Ross procedure in children and adolescents. Hellenic J Cardiol. 2006;47:337-43.

35. Bohm JO, Botha CA, Horke A, Hemmer W, Roser D, Blumenstock G, et al. Is the Ross operation still an acceptable option in children and adolescents? Ann Thorac Surg. 2006;82:940-7.

36. Hazekamp MG, Grotenhuis HB, Schoof PH, Rijlaarsdam ME, Ottenkamp J, Dion RA. Results of the Ross operation in a pediatric population. Eur $J$ Cardiothorac Surg. 2005;27:975-9.

37. Simon P, Aschauer C, Moidl R, Marx M, Keznickl FP, Eigenbauer E, et al. Growth of the pulmonary autograft after the Ross operation in childhood. Eur J Cardiothorac Surg. 2001;19:118-21.

38. Pessotto R, Wells WJ, Baker CJ, Luna C, Starnes VA. Midterm results of the Ross procedure. Ann Thorac Surg. 2001;71:S336-9.

39. Piccardo A, Ghez O, Gariboldi V, Riberi A, Collart F, Kreitmann B, et al. Ross and Ross-Konno procedures in infants, children and adolescents: a 13-year experience. J Heart Valve Dis. 2009;18:76-83.

40. Reddy VM, McElhinney DB, Phoon CK, Brook MM, Hanley FL. Geometric mismatch of pulmonary and aortic annuli in children undergoing the Ross procedure: implications for surgical management and autograft valve function. J Thorac Cardiovasc Surg. 1998;115:1255-63.

41. Luciani GB, Lucchese G, Carotti A, Brancaccio G, Abbruzzese P, Caianiello G, et al. Two decades of experience with the Ross operation in neonates, infants and children from the Italian Paediatric Ross Registry. Heart. 2014;100:1954-9.

42. Bansal N, Kumar SR, Baker CJ, Lemus R, Wells WJ, Starnes VA. Age-related outcomes of the ross procedure over 20 years. Ann Thorac Surg. 2015;99:2077-85.

43. Nelson JS, Pasquali SK, Pratt CN, Yu S, Donohue JE, Loccoh E, et al. Long-term survival and reintervention after the Ross procedure across the pediatric age spectrum. Ann Thorac Surg. 2015;99:2086-95.

44. Masuda M, Kado H, Ando Y, Shiose A, Nakano T, Fukae K, et al. Intermediateterm results after the aortic valve replacement using bileaflet mechanical prosthetic valve in children. Eur J Cardiothorac Surg. 2008;34:42-7.

45. Burczynski P, Mozol K, Mirkowicz-Malek M, Haponiuk I, Kansy A, Lipinski W, et al. Evolving approach to aortic valve replacement in children and adolescentsa preliminary report. Kardiol Pol. 2007;65:654-63.

46. Shanmugam G, MacArthur K, Pollock J. Mechanical aortic valve replacement: long-term outcomes in children. J Heart Valve Dis. 2005;14:166-71.

47. Alexiou C, McDonald A, Langley SM, Dalrymple-Hay MJ, Haw MP, Monro JL. Aortic valve replacement in children: are mechanical prostheses a good option? Eur J Cardiothorac Surg. 2000;17:125-33.
48. Lupinetti FM, Duncan BW, Scifres AM, Fearneyhough CT, Kilian K, Rosenthal GL, et al. Intermediate-term results in pediatric aortic valve replacement. Ann Thorac Surg. 1999;68:521-6.

49. Champsaur G, Robin J, Tronc F, Curtil A, Ninet J, Sassolas F, et al. Mechanical valve in aortic position is a valid option in children and adolescents. Eur $J$ Cardiothorac Surg. 1997;11:117-22.

50. Abid F, Abid A, Fekih M, Zaouali RM, Ben Ismail M. Aortic valve replacement in children under 16 years of age with congenital or rheumatic valvular disease. A study of 64 cases. J Cardiovasc Surg. 1992;33:265-71.

51. Cabalka AK, Emery RW, Petersen RJ, Helseth HK, Jakkula M, Arom KV, et al. Long-term follow-up of the St. Jude Medical prosthesis in pediatric patients. Ann Thorac Surg. 1995;60:S618-23.

52. Yamak B, Sener E, Kiziltepes U, Gol K, Tarcan O, Mavitas B, et al. Low dose anticoagulation after St. Jude Medical prosthesis implantation in patients under 18 years of age. J Heart Valve Dis. 1995;4:274-8.

53. Clarke DR, Campbell DN, Hayward AR, Bishop DA. Degeneration of aortic valve allografts in young recipients. J Thorac Cardiovasc Surg. 1993;105:934-42.

54. Ruel M, Chan V, Bedard P, Kulik A, Ressler L, Lam BK, et al. Very long-term survival implications of heart valve replacement with tissue versus mechanical prostheses in adults <60 years of age. Circulation. 2007;116:I294-300.

55. Andreas M, Wiedemann D, Seebacher G, Rath C, Aref T, Rosenhek R, et al. The Ross procedure offers excellent survival compared with mechanical aortic valve replacement in a real-world setting. Eur J Cardiothorac Surg. 2014;46: 409-14.

56. Knott-Craig CJ, Elkins RC, Santangelo KL, McCue C, Lane MM. Aortic valve replacement: comparison of late survival between autografts and homografts. Ann Thorac Surg. 2000;69:1327-32.

57. Henaine R, Roubertie F, Vergnat M, Ninet J. Valve replacement in children: a challenge for a whole life. Arch Cardiovasc Dis. 2012;105:517-28.

58. Alsoufi B, Fadel B, Bulbul Z, Al-Ahmadi M, Al-Fayyadh M, Kalloghlian A, et al. Cardiac reoperations following the Ross procedure in children: spectrum of surgery and reoperation results. Eur J Cardiothorac Surg. 2012;42:25-31.

59. Bekkers JA, Klieverik LM, Raap GB, Takkenberg JJ, Bogers AJ. Aortic root reoperations after pulmonary autograft implantation. J Thorac Cardiovasc Surg. 2010;140:S58-63; discussion S86-91.

60. Kanter KR, Kirshbom PM, Kogon BE. Redo aortic valve replacement in children. Ann Thorac Surg. 2006;82:1594-7.

61. Stulak JM, Burkhart HM, Sundt TM III, Connolly HM, Suri RM, Schaff HV, et al. Spectrum and outcome of reoperations after the Ross procedure. Circulation. 2010;122:1153-8.

62. Agarwal S, Rawtani S, Geelani MA, Moharana M, Singh H, Banerjee A. Risk factors for prosthetic valve endocarditis - a case control study. Ind J Thorac Cardiovasc Surg. 2009;25:102-6.

63. Aicher D, Holz A, Feldner S, Kollner V, Schafers HJ. Quality of life after aortic valve surgery: replacement versus reconstruction. J Thorac Cardiovasc Surg. 2011;142:e19-24.

64. Nishimura RA, Otto CM, Bonow RO, Carabello BA, Erwin JP III, Guyton RA, et al. 2014 AHA/ACC guideline for the management of patients with valvular heart disease: a report of the American College of Cardiology/American Heart Association Task Force on Practice Guidelines. J Thorac Cardiovasc Surg. 2014;148:e1-132.

65. Rippel RA, Ghanbari H, Seifalian AM. Tissue-engineered heart valve: future of cardiac surgery. World J Surg. 2012;36:1581-91.

66. Vesely I. Heart valve tissue engineering. Circ Res. 2005;97:743-55.

67. Mol A, Smits AI, Bouten CV, Baaijens FP. Tissue engineering of heart valves: advances and current challenges. Expert Rev Med Devices. 2009;6:259-75.

68. Ioannidis JP, Lau J. Pooling research results: benefits and limitations of meta-analysis. Jt Comm J Qual Improv. 1999;25:462-9.

Key Words: aortic valve replacement, pediatric, children, systematic review, meta-analysis 


\section{APPENDIX E1. LITERATURE SEARCH QUERY PubMed (1161 results)}

((()(aortic valve replacement OR ross OR (heart valve prosthesis implantation [MeSH] AND (aorta OR aortic)))) AND (allograft OR autograft OR mechanical OR prosthetic OR homograft OR bioprosthe* OR xenograft OR xenoprosthe* OR porcine OR bovine) AND (("1990/01/01"'[PDat] : “3000/12/31" [PDat]) AND Humans[Mesh] AND English [lang] AND (infant[MeSH] OR child[MeSH] OR adolescent $[\mathrm{MeSH}]))))$ )

\section{Embase (1181 results)}

(aortic AND ('valve'/exp OR valve) AND replacement OR ross) AND ('allograft'/exp OR allograft OR 'autograft'/exp
OR autograft OR mechanical OR prosthetic OR 'homograft'/exp OR homograft OR bioprosthe* OR xenograft OR xenoprosthe* OR porcine OR bovine) AND [humans]/lim AND [english]/lim AND [1990-2015]/py AND ([newborn]/ $\lim$ OR [infant]/lim OR [child]/lim OR [adolescent]/lim)

PubMed as supplied by publisher (122 results)

(()((aortic valve replacement OR ross OR (heart valve prosthesis implantation [MeSH] AND (aorta OR aortic)))) AND (allograft OR autograft OR mechanical OR prosthetic OR homograft OR bioprosthe* OR xenograft OR xenoprosthe* OR porcine OR bovine) AND (("1990/01/ 01”[PDat] : “3000/12/31”[PDat]) AND English[lang])))) AND publisher[sb] 


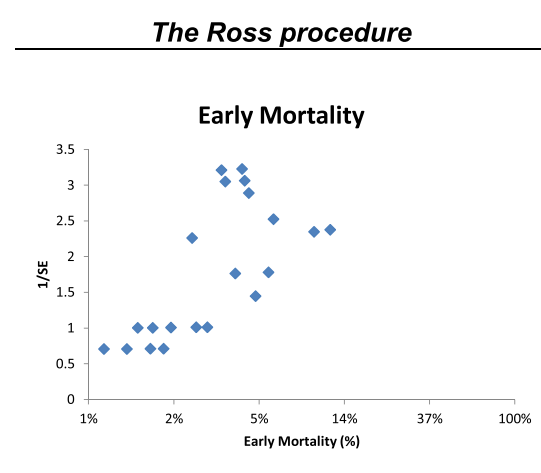

MP AVR
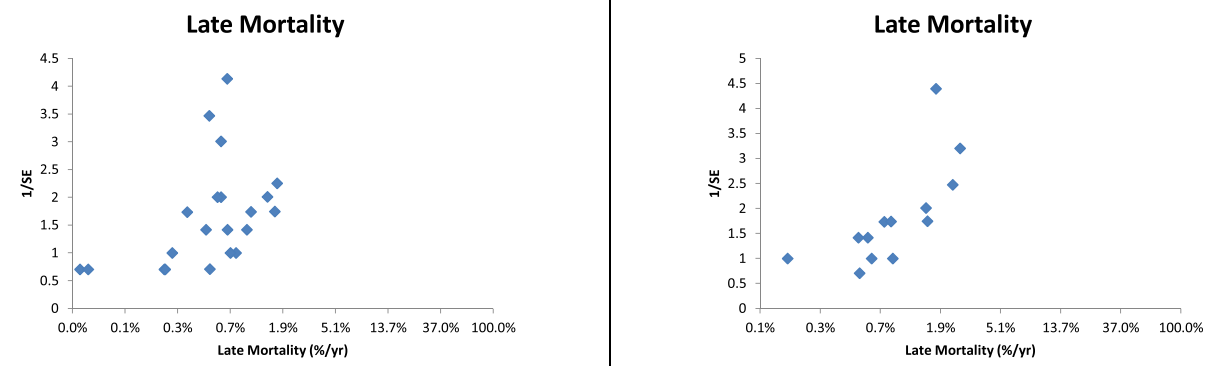

AV Reoperations
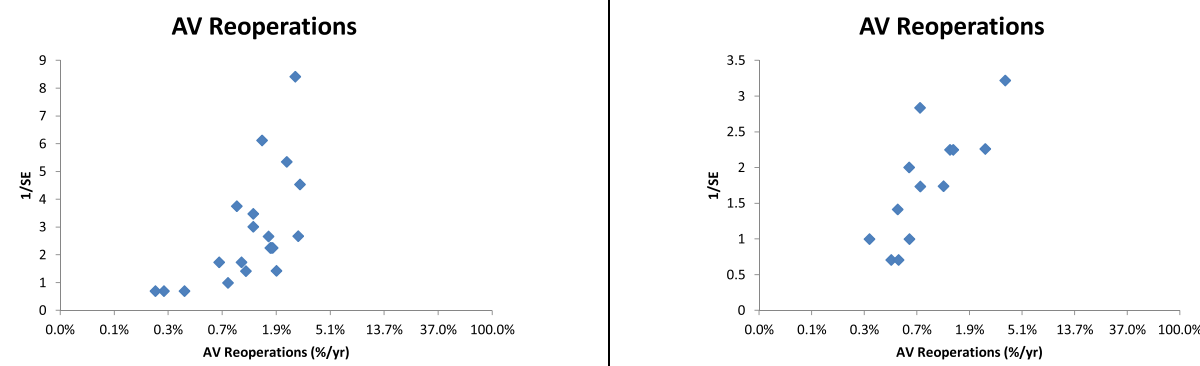

RVOT Reoperations
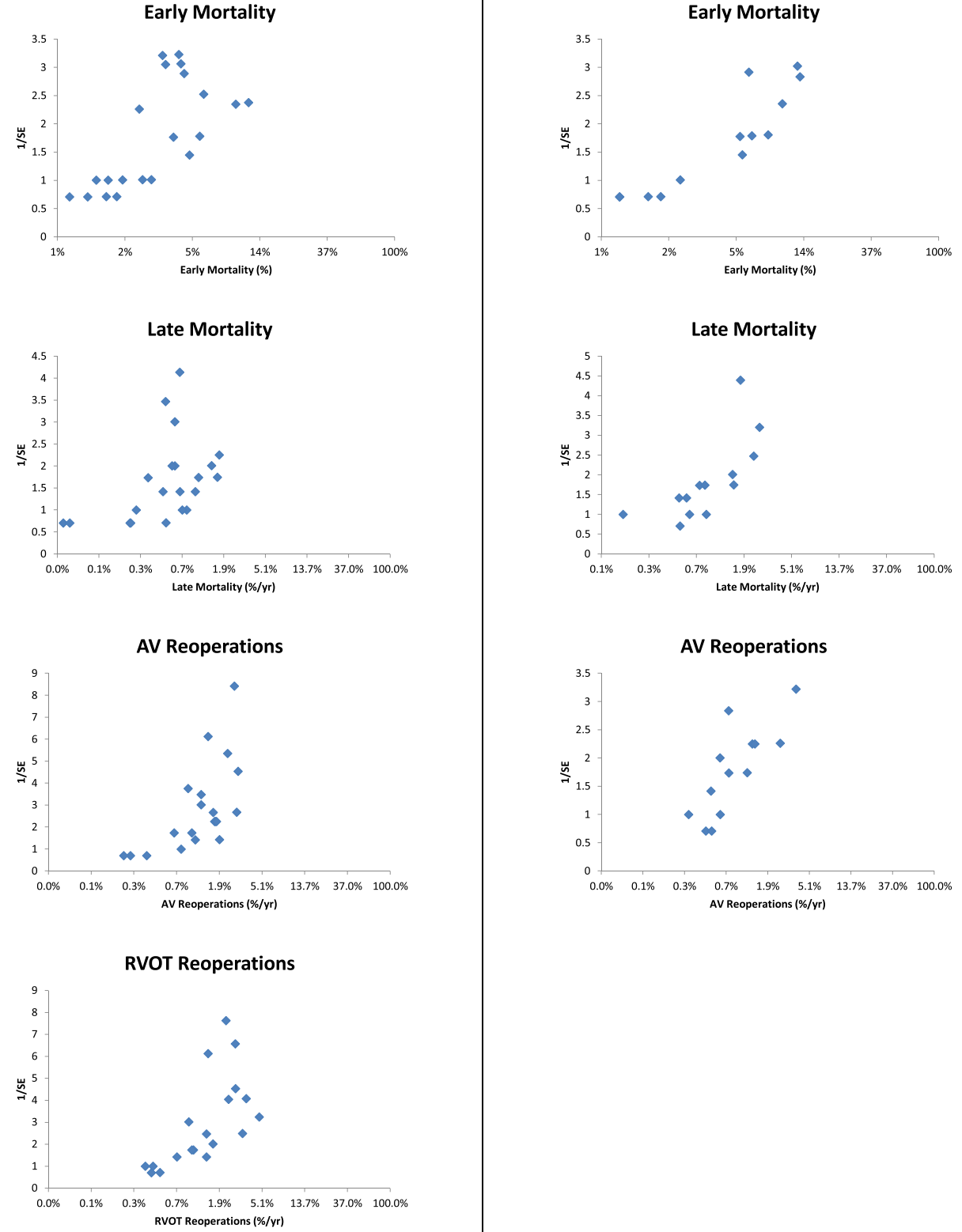

Late Mortality

FIGURE E1. Funnel plots on a natural $\log \mathrm{x}$-axis. Studies that concerned only neonates and infants were excluded from these funnel plots. Studies on homograft AVR were too few to yield conclusive funnel plots. $S E$, Standard error; $M P$, mechanical prosthesis; $A V(R)$, aortic valve (replacement); RVOT, right ventricular outflow tract; $S V D$, structural valve deterioration; $N S V D$, nonstructural valve dysfunction; $T E$, thromboembolism; VT, valve thrombosis. 


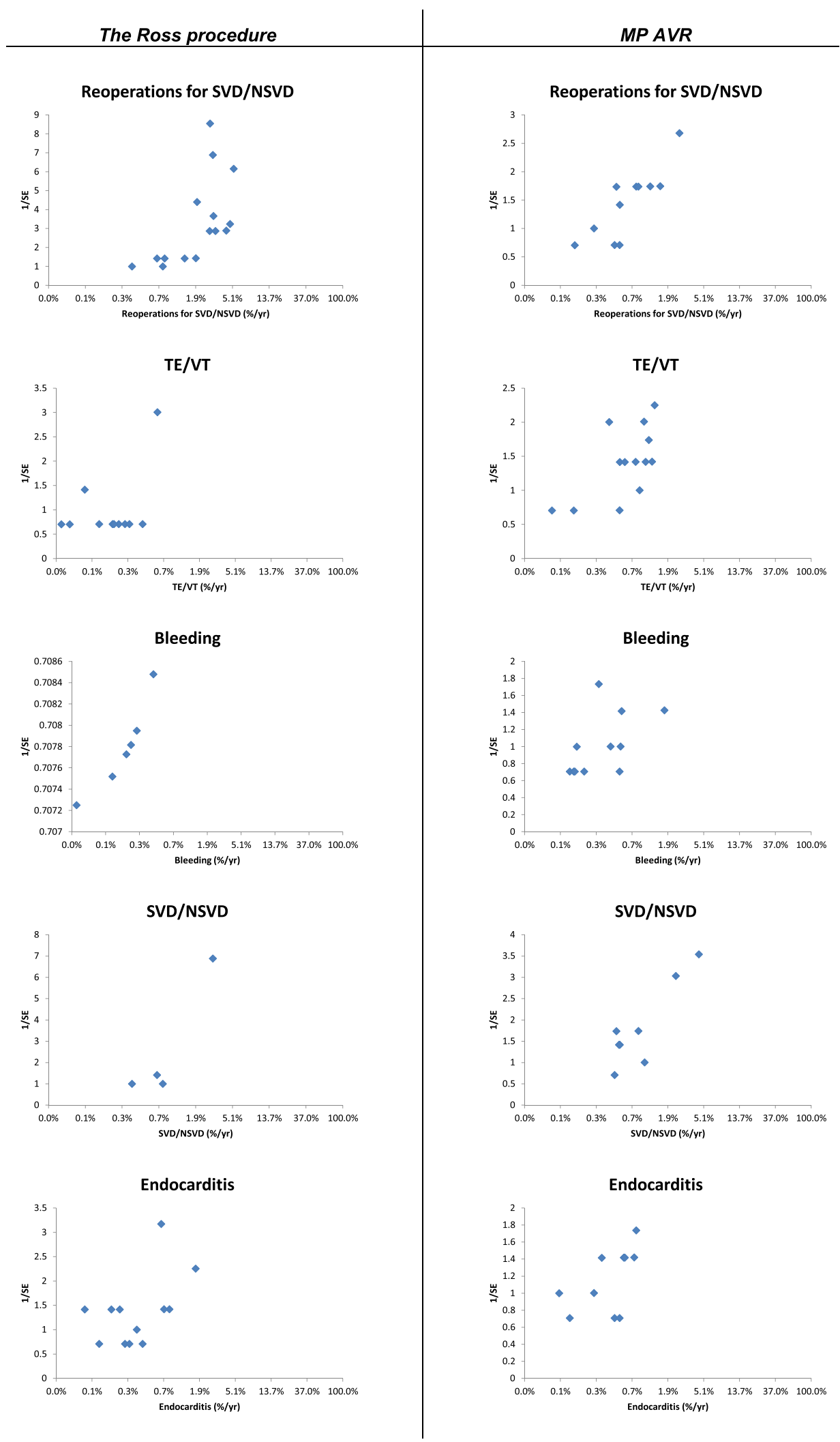

FIGURE E1. (Continued). 